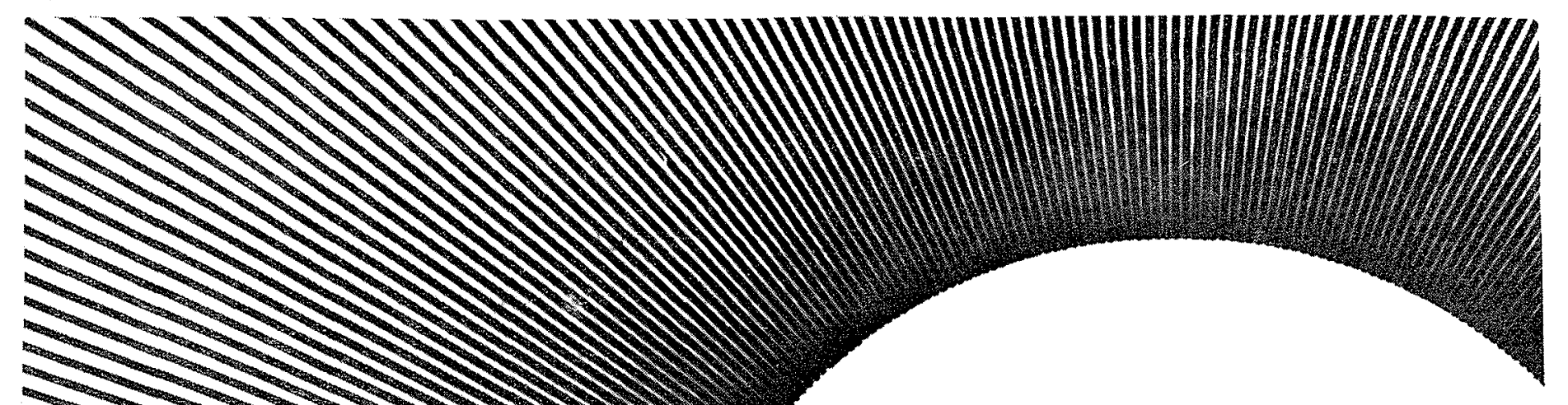

\title{
DÉTERMINATION EXPÉRIMENTALE DES FORCES HYDRODYNAMIQUES FLUCTUANTES DANS LES CUVES DU RÉACTEUR PHÉNIX
}

\author{
par P. LAMBERT et P. LEON \\ Ingénieurs au Service de Recherche Nucléaire \\ et Thermique de SOGREAH
}

\section{Introduction}

Dans le réacteur Phénix, réacteur à neutrons rapides refroidi au sodium, l'hydraulique et tous les problèmes qui en découlent, jouent un rôle important. Ce réacteur, du type intégré, est composé très schématiquement de deux grandes cuves concentriques remplies de sodium liquide dans lequel sont immergés, non seulement les parties nucléaires du réacteur (le cœur et ses protections neutroniques), mais aussi les pompes, les échangeurs et un certain nombre de structures annexes (baffles, bras de manutention, instrumentation...). Cette disposition réduit considérablement les écoulements en conduite qui sont limités au refoulement des pompes vers la base du cœur. La sortie des échangeurs et l'aspiration des pompes se font directement dans la cuve dite principale, tandis que l'alimentation des échangeurs se fait directement à partir de la cuve dite primaire. On peut suivre sur la figure 1 le schéma général de l'écoulement sortant verticalement du cœur à une vitesse moyenne de $6 \mathrm{~m} / \mathrm{s}$ dans la cuve primaire, entrant dans les échangeurs pour en sortir dans la cuve principale et être enfin aspiré par les pompes.

A la lumière de cet examen, deux faits principaux sont mis en relief

- la présence de deux surfaces libres, dont l'une (celle de la cuve primaire) est très importante;

- l'existence d'écoulements au sein même de la masse du liquide et à proximité de structures mécaniques qui sont donc soumises à des forces hydrodynamiques.
Cette double remarque est à la base du problème qui s'est posé pour l'étude sur modèle réduit (échelle $1 / 4$ ) des vibrations des structures immergées et des cuves: faut-il s'en tenir à une similitude hydraulique ne modifiant pas le nombre de Froude, à cause de la présence des surfaces libres? ou peut-on choisir une similitude hydraulique de vitesse (même vitesse sur le modèle que sur le prototype) en admettant que les excitations des structures sont indépendantes des surfaces libres?

Cette deuxième solution a l'avantage de permettre une étude directe des vibrations des structures mécaniques sur le modèle à échelle réduite: en effet, les vibrations mécaniques et les écoulements hydrauliques obéissent alors aux mêmes lois de similitude: les forces hydrauliques sont à la même échelle que les forces élastiques et les forces d'inertie des structures; il en est de même des fréquences hydrauliques et mécaniques. Ce n'est pas le cas pour la première solution, la similitude de Froude imposant soit une distorsion des dimensions géométriques des structures assimilables à des poutres, soit un changement de la nature du matériau des structures comparables à des coques minces comme les cuves du réacteur. Il est alors préférable d'utiliser une méthode indirecte qui a donc été choisie pour étudier sur modèle réduit les vibrations des cuves du réacteur, du baffle hydraulique, des cloches d'échangeurs et des cheminées d'aspiration des pompes.

La méthode indirecte consiste à mesurer sur le modèle hydraulique les excitations hydrodynamiques des structures et à analyser ensuite ces excitations afin de pouvoir calculer la réponse des structures. 


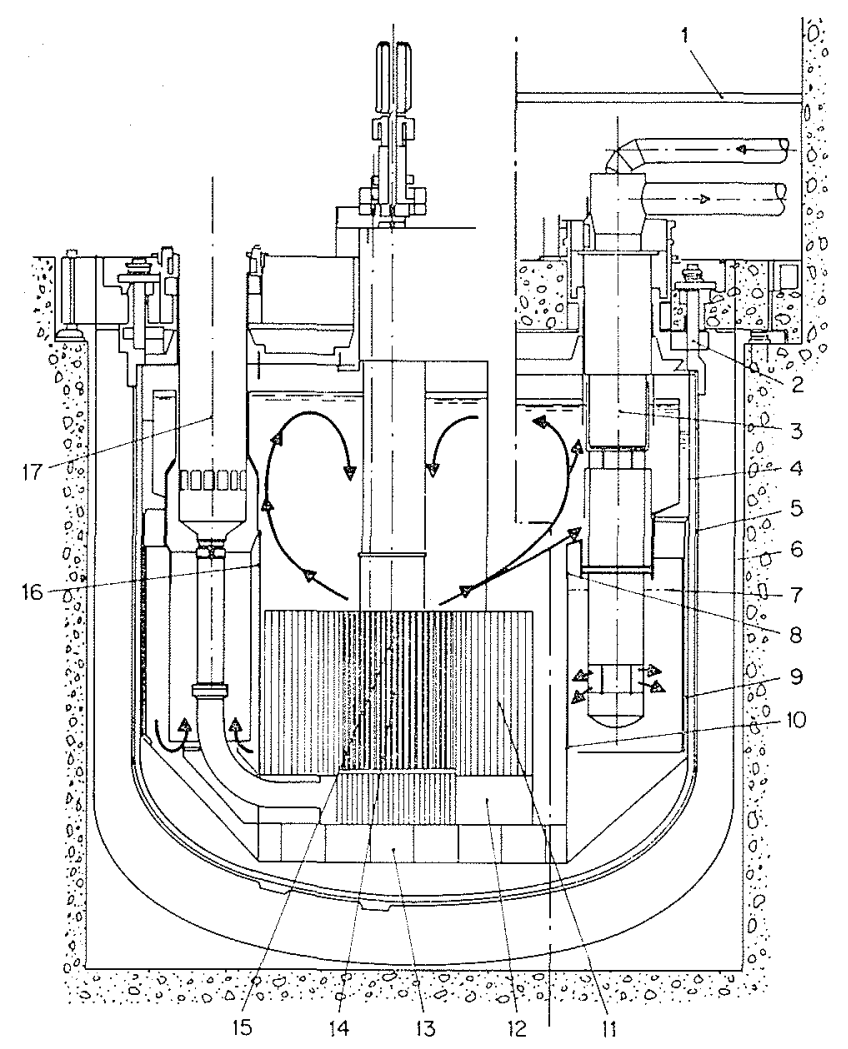

1/ Coupe schématique verticale du réacteur Phenix. 1. Plancher. 2. Suspentes. 3. Echangeur intermédiaire. 4. Cuve principale. 5. Double enveloppe. 6. Cuve d'enceinte primaire. 7. Grille. 8. Baffles de cuve primaire. 9. Baffles de cuve principale. 10. Cuve primaire. 13. Platelage 14 tronique laterale. supérieure. 16. Cuve primaire. 17. Pompe.

Si l'on désigne par $\mathrm{S}_{x}(f)$ la densité spectrale d'énergie de l'excitation et par $\mathrm{H}(f)$ le module de la fonction de transfert de la structure, la densité spectrale d'énergie de la réponse s'écrit :

$$
\mathrm{S}_{y}(f)=\mathrm{S}_{p}(f) \mathrm{H}(f)^{2}
$$

on obtient ensuite :

$$
\sigma^{2}=2 \int_{0}^{\infty} \mathrm{S}_{y}(f) d f
$$

$\sigma$ étant la valeur quadratique moyenne de la réponse de la structure.

Le problème est en fait plus complexe, les excitations pouvant être multiples. Mais la méthode reste la même et nécessite en premier lieu la mesure des excitations et leur analyse.

\section{Méthode. Appareillage Similitude}

\section{Méthode.}

Les mesures des excitations hydrauliques sur le modèle, ont été faites au moyen de capteurs de pression mesurant les fluctuations de pression en un certain nombre de points répartis sur la paroi de chacune des structures étudiées. L'emplacement des points de mesure est choisi en fonc- tion de la géométrie de la structure et de la configuration générale de l'écoulement.

Considérons par exemple la cuve primaire (fig. 1). Cette cuve est composée d'une virole cylindrique inférieure, d'une virole cylindrique supérieure et d'un redan en forme de tronc de cône reliant les deux viroles. Dix-huit points de mesure sont répartis sur la face interne de la cuve dans trois plans horizontaux: le premier plan coupe la virole inférieure dans sa partie haute, le deuxième le redan en son milieu, et le troisième, la virole supérieure à mi-hauteur de la surface libre. Dans chaque plan, les six emplacements sont déterminés en fonction des singularités qui perturbent l'écoulement, telles que les cloches d'échangeurs et les pompes.

Les pulsations de pression détectées par deux capteurs, en des points différents d'une même structura ou partie de structure, sont enregistrées simultanément sur bande magnétique à des fins d'analyse. Le calcul ultérieur de la réponse de la structure à ces sollicitations nécessite de connaître le déphasage entre les pulsations aux différents points de mesure. Pour cette raison, les deux capteurs occupent successivement les différentes positions de mesure choisies, de manièr à obtenir toutes les combinaisons deux à deux, des points de mesure sur une partie de structure. En réalité les analyses effectuées ont montré qu'il n'y avait aucune corrélation entre les pulsations de pression mesurées en deux points quelconques. Ceci nous a amenés à rechercher la longueur de corrélation, en mettant les capteurs le plus près possible l'un de l'autre, puis en les écartant progressivement. Cette longueur de corrélation est de quelques centimètres sur le modèle dont le diamètre est égal à 2.60 mètres.

\section{Appareillage.}

Les capteurs de pression utilisés sont des capteurs de pression différentielle Scientific Advances. L'élément sensible est une membrane métallique de $6,35 \mathrm{~mm}$ de diamètre, équipée de jauges à semi-conducteurs. Le capteur lui-même est incorporé à une monture de plus grandes dimensions que l'on peut fixer directement sur la structure étudiée. Un pont d'extensométrie Hottinger permet de mesurer et d'amplifier le signal détecté par le capteur, signal qui est ensuite enregistré sur papier photographique et sur bande magnétique.

La chaîne d'analyse comprend essentiellement un analyseur dynamique à filtre suiveur Spectral Dynamics, une table traçante et un générateur de balayage. Elle ne permet pas d'analyser simultanément deux signaux. On peut donc obtenir les spectres directs de densité d'énergie, mais les sceptres croisés et les mesures de corrélation ont nécessité l'utilisation d'un autre moyen d'analyse.

\section{Similitude.}

La méthode indirecte, qui a été choisie et exposée cidessus, n'impose aucun impératif quant à la similitude hydraulique, si ce n'est celui de pouvoir transposer valablement au prototype les valeurs mesurées.

Elle permet donc, dans une certaine mesure, de comparer deux similitudes hydrauliques différentes. Ceci a été fait sur le modèle de la cuve principale pour lequel la similitude de Froude ne paraissait pas s'imposer du fait de la configuration de l'écoulement. Sur ce modèle, les mesures ont donc été faites successivement dans les deux similitudes: Froude et vitesse réelle. Par contre, seule la similitude de Froude a été appliquée dans la cuve primaire où la surface libre est importante et influencée par l'écoulement principal. 


\section{ENREGISTREMENT DES FLUCTUATIONS DE PRESSION d) fluctuations globales \\ ENREGISTREMENT DESFLUCTUATIONS DE PRESSION}

\section{plenche 1}

c) fluctuations globoles

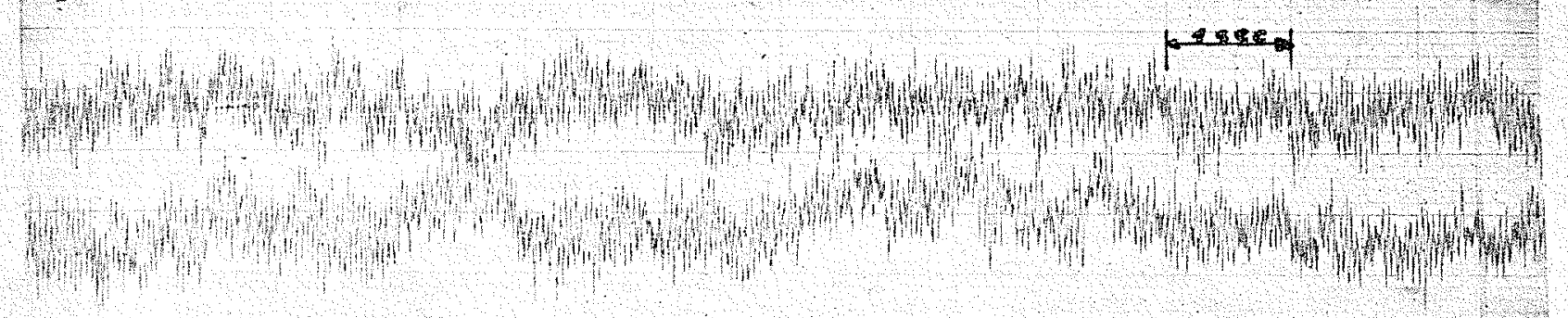

\section{b) fluctuations de basse fréquence ( $24 \mathrm{HZ}$ filtré)}

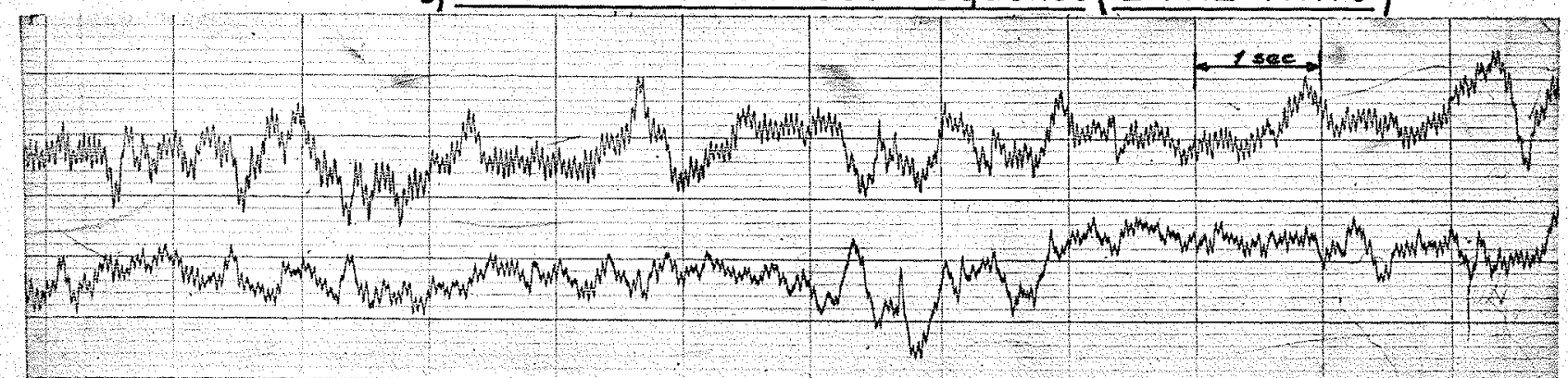

\section{Planche 1/}

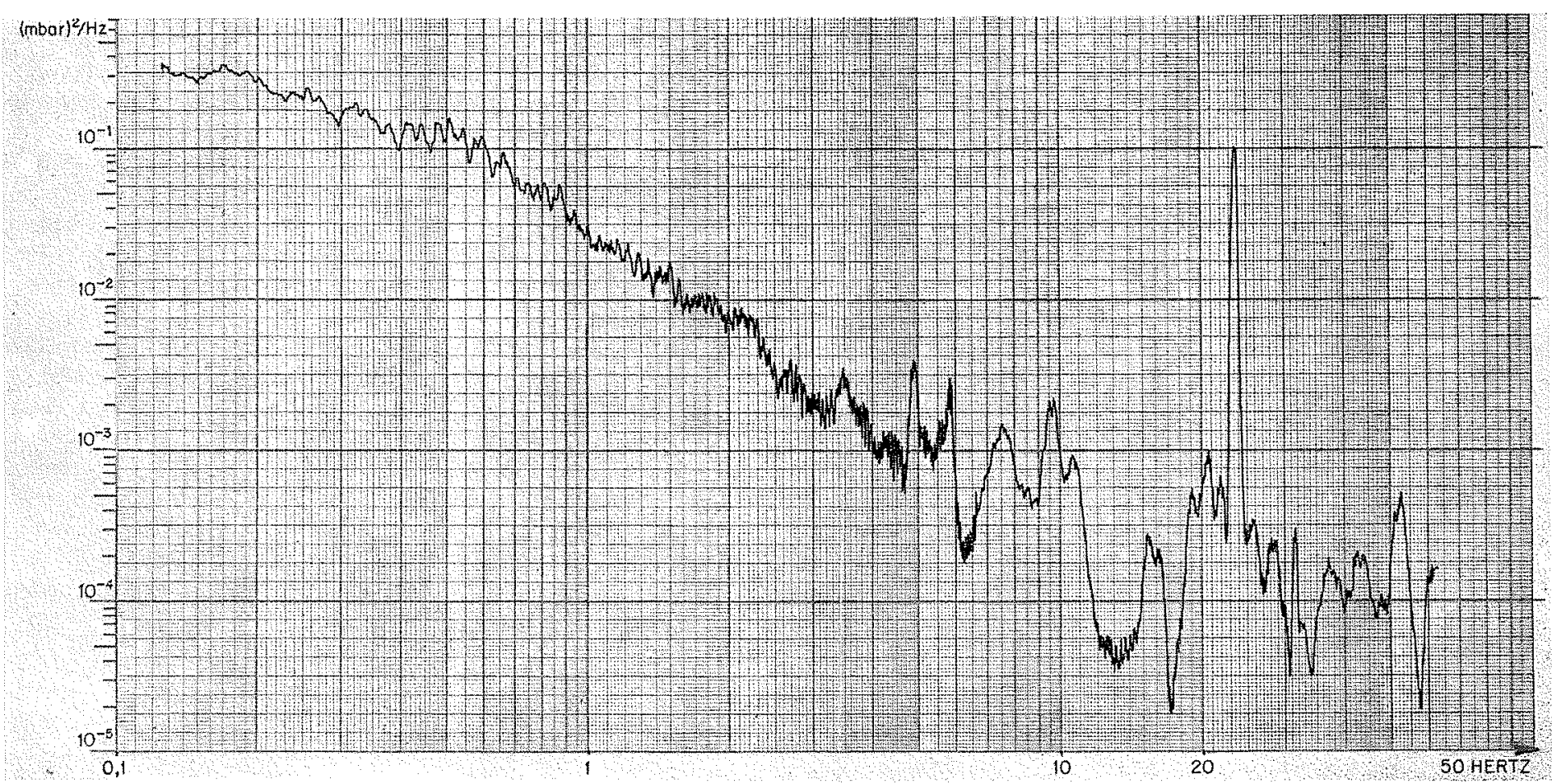

2/ Phenix. Cuve principale. Modèle 1/4. Essai 4. Spectre d'énergie. Pression P2. 


\section{Résultats et commentaires}

Les pulsations de pression mesurées ont un caractère aléatoire dans le domaine des basses fréquences inférieures à quelques $\mathrm{Hz}$. A ces fluctuations aléatoires se superposent des fluctuations périodiques de $24 \mathrm{~Hz}$. Ces dernières sont attribuées aux pompes d'alimentation des modèles dont la vitesse de rotation est de 24 tours par seconde. La planche $\mathrm{n}^{\circ} 1$ montre, d'une part la fluctuation globale, d'autre part la fluctuation aléatoire, soit après filtrage du $24 \mathrm{~Hz}$. Le niveau maximum des fluctuations aléatoires est de l'ordre de 2 millibars crête-à-crête. Ce niveau diffère très peu d'un point de mesure à un autre, sauf dans l'espace annulaire près de la surface libre de la cuve principale, c'est-à-dire assez loin de l'écoulement principal. Quand on passe d'une similitude à l'autre, c'est-à-dire quand on double le débit, ce niveau de fluctuations aléatoires n'augmente pas dans le rapport du carré des vitesses, mais plutôt dans le rapport des vitesses. Les spectres de densité d'énergie des fluctuations de pression sur la cuve primaire et sur la cuve principale ont la même allure (fig. 2). Ils sont caractérisés par:

- un pic étroit à $24 \mathrm{~Hz}$, beaucoup plus important sur la cuve principale que sur la cuve primaire où il n'apparaît pas toujours. Cette fréquence correspond à la vitesse de rotation de la pompe du modèle. Cette fluctuation périodique est amortie par la perte de charge du cœur dans la cuve primaire; elle est amplifiée par des résonances mécaniques dans la cuve principale;

- un spectre continu décroissant dans la bande de fréquences s'étendant de zéro à quelques $\mathrm{Hz}$.

Ce spectre continu est comparable au spectre de la turbulence homogène et isotrope de Taylor qui a pour équation :

$$
W=\frac{W_{0}}{1+\alpha^{2} \omega^{2}}
$$

W étant la densité spectrale d'énergie relative aux fluctuations de vitesse, $W_{0}$ et $\alpha$ sont des constantes, $\omega$ est la pulsation.

Par exemple, les densités spectrales des fluctuations de pression mesurées dans la cuve primaire sont assez bien représentées par la formule:

$$
\mathrm{W}=\frac{0,18}{1+0,02 \omega^{2}} \quad(\mathrm{mbars})^{2} / \mathrm{Hz}
$$

Comparons les spectres des fluctuations de pression mesurées dans la cuve principale en similitude de Froude d'une part, et en similitude de vitesse réelle d'autre part. Le rapport des vitesses entre ces deux similitudes étant égal à 2 , on pourrait s'attendre à un rapport de fluctuations de pression proportionnel au carré des vitesses, c'est-à-dire égal à 4 . Ceci nous conduirait à un rapport de densités spectrales d'énergie de 8 , en tenant compte du rapport des fréquences qui est égal à 2. Cette comparaison a été faite pour les fluctuations de pression mesurées sur la partie inférieure de la cheminée d'aspiration des pompes qui est très proche de la sortie des échangeurs. En fonction de la fréquence, le rapport des densités d'énergie des fluctuations entre les deux similitudes varie de 1,7 à 5,5 ; la valeur moyenne de ce rapport est égale à 3,1 . On est donc assez loin de la valeur théorique de 8 . Pour expliquer cette différence, on pourrait émettre l'hypothèse que les fluctuations de pression dépendent de la vitesse, et non du carré de la vitesse; le rapport des densités spectrales d'énergie des fluctuations serait alors égal à 2 , valeur plus proche de la valeur moyenne expérimentale.

D'une manière plus générale, il nous est apparu que quelques précautions particulières devraient être prises pour mesurer les fluctuations de pression sur modèle réduit. Ces précautions concernent la réalisation du modèle luimême mais aussi des circuits annexes (alimentation, rejets...). En ce qui concerne le modèle, il est souhaitable d'éviter toute vibration d'une structure quelconque en contact avec le fluide. En effet, ces vibrations émettent des ondes de pression se propageant dans le fuide et qui sont donc mesurées par les capteurs de pression. En ce qui concerne l'alimentation du modèle, dans l'hypothèse où cette alimentation ne peut être conforme à celle du prototype, il est souhaitable d'éviter toute source de fluctuations de pression ou de débit susceptibles de se traduire par des fluctuations parasites au niveau des capteurs.

\section{Conclusion}

Les fluctuations de pression mesurées sur le modèle réduit des cuves du réacteur Phénix ont un caractère aléatoire dans le domaine des basses fréquences.

Le niveau global des fuctuations varie très peu quand on se déplace sur la paroi des cuves du réacteur, sauf dans des zones particulières. En remplaçant la similitude de Froude par une similitude de vitesse réelle, soit en doublant les vitesses moyennes, les fluctuations de pression augmentent plutôt dans le rapport des vitesses moyennes que dans le rapport du carré des vitesses.

Les analyses spectrales de densités d'énergie confirment les conclusions précédentes; les spectres directs sont des spectres continus et décroissants dans une bande de basses fréquences ne s'étendant guère au-delà d'une dizaine de $\mathrm{Hz}$. On peut les comparer au spectre de la turbulence homogène et isotrope.

Enfin cette détermination sur modèle réduit des forces hydrodynamiques fluctuantes par la mesure des pulsations de pression exige un certain nombre de précautions dans la réalisation du modèle lui-même et dans la conception de son alimentation, afin d'éviter les fluctuations de pression parasites rendant l'analyse des résultats beaucoup plus délicate. 


\section{Discussion}

Président: M. VENDRYes

M. le Président remercie M. LÉon de son exposé et souligne la contribution apportée à ces études par SOGREAH.

La connaissance des écoulements et la prévision de leurs effets - notamment en matière de vibrations - est, dit-il, une des questions importantes que nous avons eu à résoudre à l'occasion de l'étude du réacteur Phénix; je dirai plus généralement que la compréhension de l'ensemble des phénomènes hydrauliques et thermiques à l'intérieur de la cuve principale remplie de sodium, la détermination du champ des vitesses et des températures à l'intérieur de cette cuve principale, dont la structure interne est très complexe, est certainement un des problèmes majeurs auxquels nous nous sommes heurtés à partir du moment où nous sommes passés à cette conception intégrée que vous rappeliez tout à l'heure pour les réacteurs refroidis au sodium.

Je ne peux pas dire, pour ma part, que je me sente actuellement complètement rassuré en ce sens que j'estime que nous ayons vraiment une vue tout à fait complète, claire et sûre de ces phénomènes; je crois qu'il nous faudra attendre la mise en service du réacteur lui-même et les mesures que nous pourrons faire à cette occasion pour être complètement rassurés quelles que soient la qualité et l'ampleur des études théoriques et des essais sur maquette qui ont pu être faits jusqu'ici.

J'ouvre maintenant la discution.

M. Payan apporte les précisions suivantes:

Les fluctuations de pressions parasites provenant de l'alimentation ne sont pas toujours gênantes, lorsqu'elles sont de nature acoustique et qu'on utilise les résultats de mesure au calcul de la réponse des structures en respiration par la formule de Powell qui sera exposée dans la prochaine conférence.

En effet, dans le cas de Phénix, aux fréquences de respiration des coques auxquelles nous nous intéressons, les ondes acoustiques sont pratiquement en phase sur tout le pourtour de ces coques, compte tenu de la grande vitesse du son dans le sodium. Elles produisent des alternances de compression et d'extension uniformes de ces coques, avec des déformations et contraintes très faibles, mais ne peuvent donner les vibrations de respiration que nous craignons. Dans la formule de Powell, le fait que les ondes soient en phase se traduit par une densité cospectrale de l'excitation constante dans l'espace, qui donne bien une réponse en respiration nulle. (M. Livolant, avec qui nous avons étudié les problèmes acoustiques pourra vous donner quelques précisions).

Sur une question de M. Coursimault, M. Payan confirme que la vitesse de propagation des ondes dans le liquide est telle que celles-ci aux basses fréquences sont pratiquement en phase aux différents points d'une même coque de Phénix.

M. Livolant (C.E.A., Gif-sur-Yvette) intervient en ces termes : Dans les volumes annulaires entre les cuves primaires et principales du réacteur à sodium liquide Phénix, compte tenu du périmètre moyen, de la vitesse du son, et des caractéristiques mécaniques des structures, on peut considérer qu'il y a, en général, un ordre de grandeur de différence entre les fréquences propres des modes radiaux mécaniques des cuves et des modes acoustiques correspondants. Aux fréquences où les modes acoustiques sont amplifiés, ils n'ont donc pas d'effets mécaniques sur les cuves. Cependant, ces modes peuvent être excités, même à fréquence basse par des sources d'excitation extérieures, telles que les pompes (avec une amplitude proportionnelle à :

$$
\frac{1}{1-\left(\frac{v^{m}}{v}\right)^{2}}
$$

où $\nu_{m}$ est la fréquence du mode, et $\nu$ la fréquence d'excitation). $\mathrm{Si}$ ces sources sont suffisamment intenses, et leur fréquence proche de celle d'un mode mécanique, il peut donc y avoir excitation de ce mode mécanique par l'intermédiaire du mode acoustique.

En pratique, dans le cas de Phénix, ce phénomène paraît négligeable; des calculs en cours permettront de s'en assurer.

Répondant à M. Evenpoel, M. LÉon déclare :

Je ne pense pas qu'on puisse mesurer la réponse des structures sur le modèle - plus ou moins en similitude de Froude - parce qu'il faudrait que l'échelle des fréquences propres mécaniques soit la même que l'échelle des fréquences d'excitation hydraulique. En similitude de vitesses réelles, ceci devrait être possible, mais exigerait la réalisation d'un modèle strictement en similitude géométrique et cela pose des problèmes technologiques (réalisation des fixations et d'un certain nombre de détails).

Ayant concentré votre attention sur les excitations de fréquence relativement faibles, ne risquez-vous pas de négliger le problème le plus important qui semble l'excitation provoquée par les pompes et les moteurs se trouvant à l'intérieur de la cuve? demande M. FABRIS.

Le danger des fréquences très faibles d'excitations n'est pas négligeable, répond $M$. LÉON, particulièrement sur la virole supérieure primaire dont la fréquence propre fondamentale est de l'ordre de $0,7 \mathrm{~Hz}$; cette fréquence propre tombe bien dans le spectre de fréquence d'excitation qui a été mesuré. D'ailleurs, à la suite des études auxquelles il a contribué, le constructeur a modifié la géométrie de cette virole supérieure en renforçant le bord supérieur pour augmenter sa fréquence propre. Donc, pour les structures de très grandes dimensions, les excitations de basse fréquence sont également à craindre.

Pour ce qui est de l'excitation des vibrations par la pompe, le problème n'a pu être étudié sur modèle, celui-ci ne reproduisant pas exactement le système de l'alimentation.

On peut dire que les pompes émettent des ondes acoustiques dont les fréquences sont égales à la fréquence fondamentale et au produit de cette fréquence par le nombre de pales; on retombe donc sur le problème des fréquences acoustiques qui a été évoqué tout it l'heure. D'autre part, les pompes ont été essayées sur une maquette à échelle 1 et des mesures de fluctuations et d'excitation des pompes vont être faites afin d'avoir une idée de l'importance de ces excitations.

M. le Président clôt la discussion en remerciant les auteurs de la communication et les personnes qui ont participé à sa discussion. 


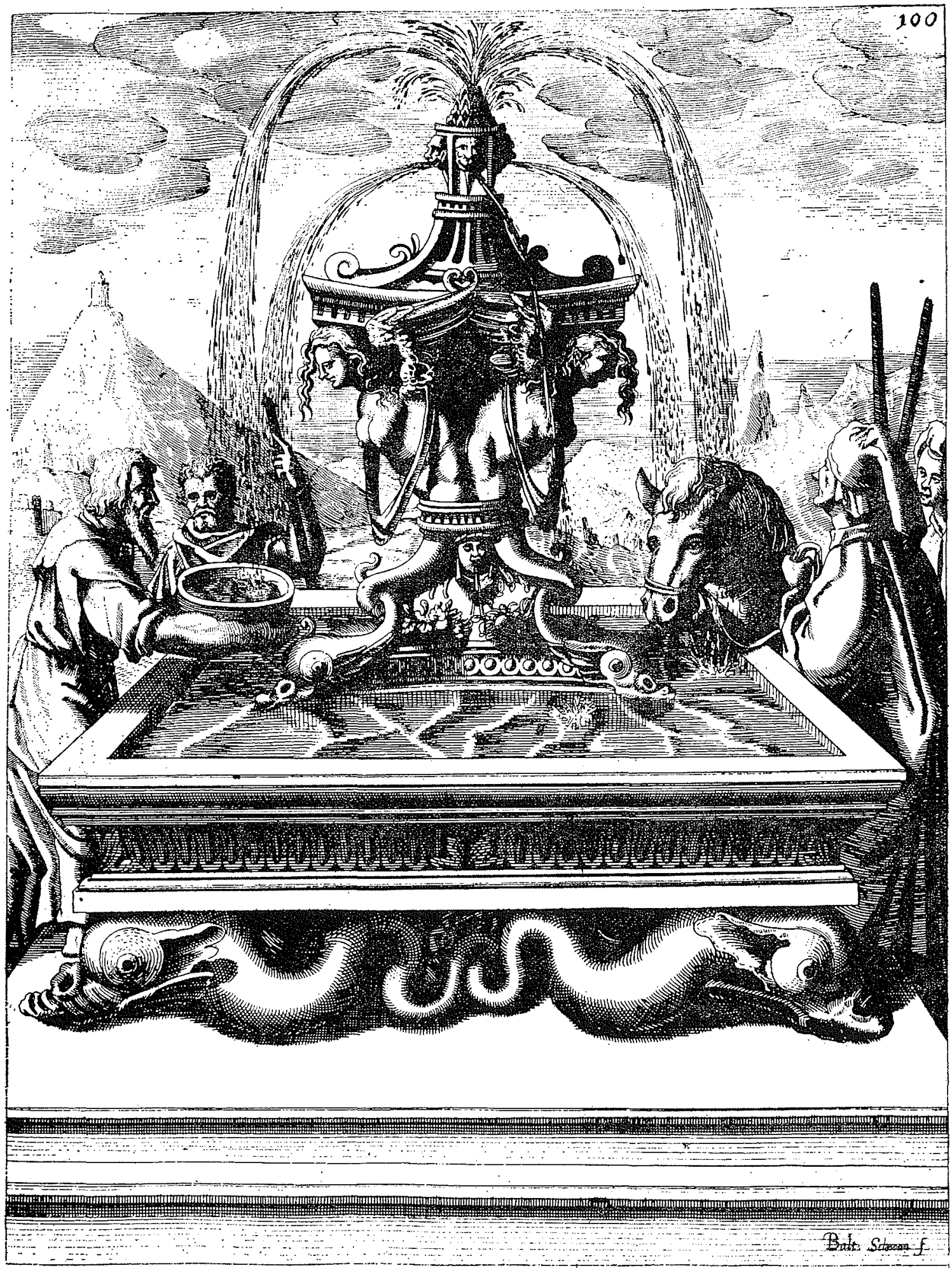

